\title{
RECONSTRUCTIVE SURGICAL CORRECTION OF CLAW HAND DEFORMITIES IN POST HANSEN DISEASE PATIENTS BY LASSO PROCEDURE.
}

\section{Dr Sumant Kumar Singh}

Dr Arun kumar*
Assistant Professor , Department Of Physical Medicine \& Rehabitalation, Pmch, Patna

Hod \& Associate Professor, Department Of Physical Medicine \& Rehabitalation, Pmch, Patna *Corresponding Author

\section{Dr Poonam Singh}

Junior Resident, Department Of Forensic Medicine \& Toxicology , Pmch, Patna

ABSTRACT The outcome of 18 claw hands deformities, corrected with the Zancolli lasso procedure was evaluated in present study, deformity, power, movement, and function of the hands were evaluated before and after surgery.The mean duration of surgery was 60 (range, 45-75) minutes. The mean duration of follow-up was 7.5 (range, 3-12) months. Deformity correction was good in 13 patients, fair in 3 , and poor in 2 . The mean angle improvement was maximum in the little finger,followed by the ring, index, and middle fingers. The Zancolli lasso procedure is easy to perform and does not require extensive postoperative training. It restores synchronous finger flexion and spares other superficialis tendons, thus avoiding swan neck deformity of the fingers.

\section{KEYWORDS : Leprosy; Fds; Zancolli Lasso; Claw Hand.}

\section{INTRODUCTION:}

India has the highest number of new leprosy cases in the world, followed by Brazil and Indonesia. Every year, over 200,000 such cases are detected globally and India accounts for more than half of these, according to the World Health Organization (WHO)Feb 28, 2019. In India, there are nearly 4 million leprosy patients, accounting for one-third of leprosy patients worldwide. The ulnar nerve at the elbow is most commonly involved and may lead to clawing of the fingers, particularly the ring and little fingers (partial claw hand), causing instability, incoordination, imbalance, and asynergism. Correction of the deformity involves prevention of hyperextension at the metacarpophalangeal joint so that normal extensors can extend the interphalangeal joints and initiate flexion of the proximal phalanx of the fingers. This study evaluated the outcome of 18 claw hands deformities corrected with the Zancolli lasso procedure.

\section{MATERIALS AND METHODS}

STUDY PERIOD: Between January 2017 and December 2019.

\section{PLACE OF STUDY: PMR department PMCH Patna.}

\section{SELECTION OF PATIENT:}

18 patients aged 15-60 (mean, 38) years with claw hand deformity of the dominant $(n=11)$ or non-dominant $(n=7)$ hand who had no infective focus in the fingers and completed multi-drug treatment for a minimum of 12 months period and underwent the Zancolli lasso procedure, in which the flexor digitorum superficialis (FDS) of the middle finger was divided in to 4 slips (one for each finger) and reattached to itself after passing through the proximal pulley.

\section{TYPE OF ANAESTHESIA:}

Brachial block/intravenous regional anaesthesia with tour niquet control was used.

\section{SURGICAL PROCEDURE:}

A transverse incision was made at the level of the distal palmer crease. Care was taken to avoid the neurovascular bundles. The flexor tendons were exposed using sharp dissection. The flexor tendon sheaths were exposed from the middle of the metacarpal to the middle of the proximal phalanx. The proximal pulley was identified by its thick, glistening fibrous strands. The flexor tendon sheath was opened proximal to the pulley by making a T-shaped incision.
Distally the digital fibrous tunnel was opened in an L-shaped incision at the level of the proximal arciform ligament.The flexor tendons were identified through the distal opening. The flexor digitorum superficialis tendon of the middle finger was hooked up and cut as distally as feasible without injuring the profundus tendon that lies beneath it. The distal cut end was allowed to retract. The tendon was withdrawn through a small curved incision at the base of the palm along the thenar crease. The tendon was split into 4 slips, one slip for each finger (Fig.1).

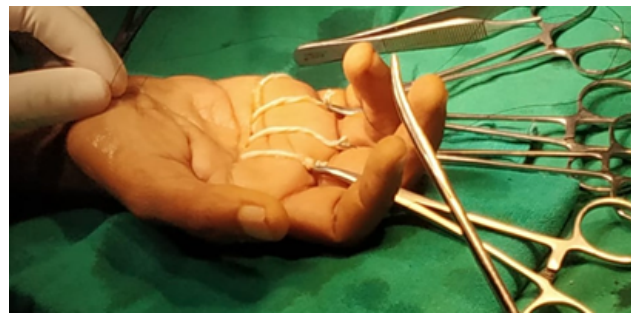

Figure : The 4 slips of the flexor digitorum superficialis of the middle finger.

The slips were passed deep to the palmar aponeurosis along the flexor sheath with the help of a tendon tunneller. The slips were then passed under the proximal pulley of the corresponding finger and through the opening distal to the pulley, and the tendon was taken out and brought palmar to the pulley and proximally. The slip was sutured to the same slip (thus forming a lasso) under proper tension with the metacarpophalangeal joint in $20^{\circ}$ to $30^{\circ}$ flexion and the wrist in $30^{\circ}$ flexion. Any excess tendon slip was cut off. This procedure was performed for all 4 fingers starting from the index finger, using the flexor digitorum superficialis of the middle finger. After obtaining complete haemostasis, the wound was closed and dressed, and anterior and posterior below-elbow plaster of Paris (POP) slab was applied with the metacarpophalangeal joints in $60^{\circ}$ to $70^{\circ}$ flexion and the wrist in $20^{\circ}$ flexion, leaving the interphalangeal joints free.

\section{POSTOPERATIVE CARE:}

Active flexion of the interphalangeal joints was allowed after 24hours. as the pain and swelling subsided. The sutures and anterior POP slab were removed on day 14, where as POP metacarpophalangeal posterior slab was applied for 4-6 weeks. during this period active flexon of finger is allowed. 
Active and passive flexion and extension exercises were started after 6weeks.

\section{REHABILITATION:}

Rehabilitation is designed to ensure that the surgical procedures undertaken can help patients to achieve their full physical potential. Preoperative strengthening of tendon of middle finger (operative hand) and proper training of tendon of hand with re education can improve the function of hand, Hands must be protected from injury and infection in order to prevent impairment and disability.

\section{FOLLOW UP:}

Patients were assessed before and after operation and at 6 weeks, 3 months, 6 months, and one year. Deformity of the hand was assessed during follow-up. The distal metacarpal arch angle was measured using a goniometer. Deformity correction was considered good, fair, and poor, respectively, when the metacarpophalangeal angle was $30^{\circ}$ to $0^{\circ}, 0^{\circ}$ to $-20^{\circ}$, and $>-20^{\circ}$, the interphalangeal angle was $0^{\circ}$ to $20^{\circ}, 21^{\circ}$ to $40^{\circ}$, and $>40^{\circ}$, and the distal metacarpal arch was $\geq 15^{\circ}$, $10^{\circ}$ to $14^{\circ}$, and $<10^{\circ}$. Grip and pinch strength were measured. And Strength was graded as good, fair, and poor when increased, unchanged, and decreased, respectively. Motion of the fingers was checked using pulp-to-pulp pinch and motion of the thumb with all the fingers. The function of the hand was assessed by the time taken to count 20 beads using the thumb and other fingers and the ability to hold a ball with a diameter of $7.5 \mathrm{~cm}$ in a normal fashion. Functional outcome was graded as good, fair, and poor.

\section{RESULTS:}

The mean duration of surgery was 60 (range, 45-75) minutes. The mean duration of follow-up was 7.5 (range, 3-12) months. Deformity correction was good in 13 patients, fair in 3 , and poor in 2.The mean angle improvement was maximum in the little finger, followed by the ring, index, and middle fingers. The transversemetacarpal arch (compared with the normal hand) was increased/reversed in 5 patients and decreased in 13 patients. The normal angle in the open hand position was $20^{\circ}$ to $30^{\circ}$. The grip and pinch strength increased in 11 patients, remained unchanged in 6, and decreased in one. All hands had decreased grip and pinch strength during the postop erative 6 weeks, but after 6 months, the grip strength increased to a mean of $11 \mathrm{~kg}$ (from $9.7 \mathrm{~kg}$ preoperatively), whereas the pinch strength increased by a mean of $1.3 \mathrm{~kg}$. The mean time taken to count 20 beads using the thumb and other fingers was 60 (range, 30-90) seconds before surgery and 45 (range, 20-70) seconds after surgery, with 16 patients able to hold and count beads with pulp-to pulp pinch. 13 patients could hold a ball in a normal fashion. Motion and function of the hand was good in 11 patients, fair in 5 , and poor in 2 .

\section{DISCUSSION AND CONCLUSION}

In Hansen's disease, Ulnar nerve paralysis results in loss of synchronous digital flexion function and grasp and pinch power. Surgical procedures for correction of clawing include tendon transfers (such as the Bunnell procedure [the flexor digitorum superficialis is passed through the lumbrical canal and attached to the extensor expansion], the Brand procedure [extensor to flexor four-tailed tendon transfer], and the Antia procedure [palmaris longus four tailed tendon transfer]), tenodesis, capsulodesis, and bone block. The transferred tendon is sutured to the lateral slip of the extensor expansions and tends to lead to swan neck deformity in hypermobile fingers. The Zancolli procedure prevents hyperextension of the metacarpophalangeal joint by plicating the anterior capsule and keeping the joint at $20^{\circ}$ flexion,but it cannot achieve active initiation of flexion of the proximal phalanx. The Zancolli procedure is modified by advancing the pulley and keeping the joint at $60^{\circ}$ of flexion, using a longitudinal incision that results in flexion contracture. The Swamy and
Rao procedure excises the distal part of the palmar plate and advances and sutures the remaining portion with a single horizontal mattress suture to prevent hyperextension of the matacarpophalangeal joint. Many of these procedures are technically difficult and necessitate extensive postoperative training.Complications such as median nerve compression, stiffness of fingers, FDS minus deformity, checkrein deformity ، and under correction are high. The Zancolli lasso procedure is performed through only one incision in the palm under brachial block anaesthesia at tertiary health centre's like PMCH Patna, The procedure restores synchronous digital flexion and improves grip and pinch strength by sparing other superficial tendons (thus avoiding swan neck deformity of the fingers), the proximal inter phalangeal joint and essential for grip. In contrast, other procedures re-route the flexor digitorum superficialis to extensor expansion and weaken the already weakened hand. As the deformity improves, the grip strength increases, as the hand can be put in a more mechanically advantageous position to use the remaining muscle tendon units. The procedure also tends to correct transverse metacarpal arch. As the transverse metacarpal arch corrects, 4 -finger pinch improves. The maximum deformity improvement was noticed in patients with shorter duration of paralysis. Correct tension during suture of transferred slips and adequate protection and training of the hand during the postoperative period are important for good outcome.

\section{REFERENCES:}

1. World Health Organization-Weekly epidemiological records. Geneva: World Health Organization. 2005;80:118-24.

2. Leprosy Elimination Alliance.Leprosy elimination India: Bulletin of leprosy eliminationalliance. 2005:5:8.

3. Ministry of Health, Government of India. National leprosy eradication programme: current leprosy situation in India. Report of the Office of DGHS Central Leprosy Division. 2005

4. Job CK. Pathology of leprosy. In: Hastings RC, ed. Leprosy. Second ed. Edinburgh: Churchill Livingstone, 1994:193-232.

5. Job CK. Nerve in reversal reaction. Indian J Lepr 1996;68:43-7.

6. Ridley DS, Jopling WH. Classification of leprosy according to immunity: a fivegroup system. Int J Leprosy Other Mycobact Dis 1966;34:255-73.

7. Croft RP, Nicholls PG, Steyerberg EW, et al. A clinical prediction rule for nervefunction impairment in leprosy patients. Lancet 2000;355:1603-6.

8. Zancolli EA. Claw-hand caused by paralysis of the intrinsic muscles: $\alpha$ simple surgical procedure for its correction. J Bone Joint Surg Am 1957;39:1076-80.

9. Palande DD. Correction of intrinsic-minus hands associated with reversal of the transverse metacarpal arch. J Bone Joint Surg Am 1983;65:514-21.

10. Siddallingaswamy MK, Rao KS. Surgical correction claw fingers in Hansen's disease by palmar plate shortening and pulley advancement. J Hand Surg Am 1991;16:814-9.

11. Burkhalter WE, Strait JL. Metacarpophalangeal flexor replacement for intrinsic-muscle paralysis. J Bone Joint Surg Am 1973;55:1667-76.

12. Bunnell S. Surgery of intrinsic muscles of hand other than those producing opposition of the thumb. J Bone Joint Surg Am 1942;24:1-31.

13. Brand PW. Paralytic claw hand; with special reference to paralysis in leprosy and treatment by the sublimis transfer of Stiles and Bunnell. J Bone Joint Surg Br 1958;40:618-32.

14. Antia NH. The palmaris longus motor for lumbrical replacement. Hand 1969;1:139-45.

15. Brown PW. Zancolli capsulorrhaphy for ulnar claw hand. Appraisal of fortyfour cases. J Bone Joint Surg Am 1970;52:868.

16. Lakhan Pal VP, Yadav SS, Nair MN. Reconstructive surgery of claw hand in leprosy. Lepr India 1979;51:213-7. 